\title{
ZWEI NEUE METACYCLOPS (CRUSTACEA COPEPODA) VON DEN WESTINDISCHEN INSELN BARBADOS UND ARUBA: $M$. AGNITUS N. SP. UND M. MUTATUS N. SP., SOWIE EIN BESTIMMUNGSSCHLÜSSEL FÜR DAS GENUS
}

\author{
von \\ HANS-VOLKMAR HERBST \\ Distelweg 1, 4150 Krefeld 29 - Hüls, Bundesrepublik Deutschland
}

\begin{abstract}
In wells of the islands of Barbados and Aruba (West Indies) two new species of Metacyclops were found. One, belonging to the minutus-group, is described as Melacyclops agnitus n. sp. and is closely related to Metacyclops minutus (Claus, 1863). In absence of complete descriptions of $M$. minutus, especially concerning absolute measurements, these are completed with observations on a West-German population. The other species, called Metacyclops mutatus n. sp., may have evolved from mendocinus-like ancestors, but differs obviously in comparison with the morphologically similar species $M$. aequatorialis Dussart, 1977 and $M$. chelazzi Dumont, 1981. A key for Metacyclops is provided, mainly based on the literature, as an aid for future investigations.
\end{abstract}

\section{ZUSAMMENFASSUNG}

Aus Brunnen der Inseln Barbados und Aruba (Westindische Inseln) wurden zwei neue Metacyclops-Arten beobachtet. Eine davon gehört in die minutus-Gruppe und wird aufgrund spezifischer Merkmale, die sie von Metacyclops minutus (Claus, 1863) unterscheiden, als Metacyclops agnitus n. sp. beschrieben. Da Beschreibungen von $M$. minutus mit umfassenden Maßangaben fehlen, werden diese für eine westdeutsche Population (Raum Bonn) geliefert. Die zweite neue Art dürfte von mendocinus-ähnlichen Vorfahren abstammen. Gegenüber den morphologisch Vergleichbaren $M$. aequatorialis Dussart, 1977 und $M$. chelazzi Dumont, 1981 läßt sich die als Metacyclops mutatus n. sp. bezeichnete neue Art eindeutig abgrenzen. Als Hilfsmittel

* Report 53 appeared in Stygologia vol. 3 no. 3: 241-251 (1987). The fieldwork during which the material, described in the present paper, was collected, has been financially supported by the Beijerinck-Popping Fonds (Amsterdam), the Treub Maatschappij (Utrecht), and the Netherlands Foundation for the Advancement of Tropical Research (WOTRO, The Hague). für künftige Untersuchungen ist überwiegend aufgrund von Literaturangaben ein Bestimmungsschlüssel für die Gattung Metacyclops aufgestellt worden.

Metacyclops agnitus n. sp.

(Abb. 1-25)

Material. - Holotypus: 1 \&. Amsterdamer Expeditionen zu den Westindischen Inseln, Sta. 78-364, Barbados: Parish of Christ Church, W. von Hopewell, offener, rechteckiger Brunnen von 1,5 m Durchmesser; Wasserspiegel in 12,5 m Tiefe, 0,1 m Wasserstand; Position $13^{\circ} 03^{\prime} 23^{\prime \prime} \mathrm{N} 59^{\circ} 31^{\prime} 28^{\prime \prime} \mathrm{W}$; 7. Juni 1978; Paratypen 15 ८९, 9 ণ゚ (Zoölogisch Museum Amsterdam).

Weitere Fundorte: Sta. 78-365, Barbados, Parish of St. Philip. Oldbury Factory, geschlossener, runder Brunnen von 2,2 m Durchmesser mit zerstörter Windpumpe; Wasserspiegel in $24,5 \mathrm{~m}$ Tiefe, $5 \mathrm{~m}$ Wasserstand; Position

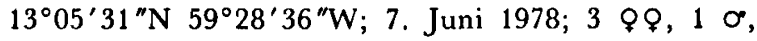
1 Copepodid (ZMA).

Beschreibung. - Das Weibchen besitzt bei einer Gesamtlänge zwischen 550 und $600 \mu \mathrm{m}$ ohne die Endborsten der Furka, eine normal cyclopoide Gestalt (Abb. 1). Das Cephalothoraxsegment nimmt etwa $60 \%$ des Thorax ein, dessen größte Breite mit rund $60 \%$ der Länge am Ende des Cephalothorax liegt.

Das Abdomen wird durch ein relativ kräftiges Genitalsegment gekennzeichnet (Abb. 2), das fast so breit wie lang ist und sich bis zum Hinterrand um etwa $30 \%$ verschmälert. Die Breite der drei folgenden Abdominalsegmente (Abb. 3) ist deutlich geringer, von ihnen ist das 3. Abdominalsegment am kürzesten, das Analsegment am längsten. Die absoluten und relativen Maße der Abdominalsegmente sind: 
Länge: Breite Maße auf das Analsegm. $=1$ berechnet (in Klammern relative Maße eines 2. Weibchens)

\begin{tabular}{|c|c|c|}
\hline enitalsegm. & 99 & $\begin{array}{r}: 95 \mu \mathrm{m}=2,48: 1,79 \\
(2,39):(1,68)\end{array}$ \\
\hline Abd. & 27 & $: 59 \mu \mathrm{m}=\begin{array}{c}0,68: 1,11 \\
(0,80):(1,05)\end{array}$ \\
\hline $\mathrm{Dad}$ & & $=$ \\
\hline
\end{tabular}

3. Abd. segm. $23: 57 \mu \mathrm{m}=0,58: 1,08$

Analsegm.

$40: 53 \mu \mathrm{m}=1 \quad: 1$

Das Receptaculum seminis (Abb. 2) besitzt eine mehr oder weniger kreisrunde Form und nimmt den größten Teil der Ventralseite des Genitalsegments ein. Die Hinterränder aller Abdominalsegmente sind glatt. Der Hinterrand des Analoperculums befindet sich etwa in der Mitte des Analsegments und ist fast gerade.

Die Furkaläste (Abb. 4) sind $3 \frac{1}{3}$ mal so lang wie in Höhe der Lateralrandborste breit. Diese steht etwas hinter der Mitte des Furkalastes (etwa 55\% der Furkalänge). Die beiden mittleren Endborsten sind lang, befiedert, die laterale von ihnen erreicht $2 / 3$ der Länge der medialen. Die schwach befiederte mediale Apikalborste ist sehr dünn und um 2/5 kürzer als die laterale. Die Dorsalborste inseriert etwa mediad gestellt, in der Mitte zwischen der Lateralrandborste und dem Ende des Furkalastes; sie ist knapp so lang wie die laterale Apikalborste. Maße der Furka und ihrer Anhänge sind: Länge : Breite $=(35+30): 19 \mu \mathrm{m}=(1,84+1,58): 1^{* *} ;$ Endborsten von außen nach innen $=45: 156: 238$ : $26 \mu \mathrm{m}=1: 3,47: 5,29: 0,58$; Dorsalborste $=$ $40 \mu \mathrm{m}=0,90: 1$; Lateralrandborste $=19 \mu \mathrm{m}=$ $0,42: 1$ (alle Verhältnismaße auf die laterale Apikalborste $=1$ bezogen).

Die 11-gliedrige 1. Antenne (Abb. 5) reicht zurückgelegt nicht bis zum Ende des Cephalothorax. Ihre mittleren Gliedlängen sind an der Basis beginnend $=40: 9: 21: 12: 7: 17: 31$ : $26: 12: 17: 23 \mu \mathrm{m}$. Die viergliedrige 2 . Antenne (Abb. 6) ist am 3. Glied mit 5 und am Endglied mit 8 Borsten bewehrt. Die Mandibel (Abb. 7) trägt auf dem rudimentären Taster

** Das + Zeichen in der Längenangabe der Furka gibt die Insertion der Lateralrandborste an. zwei lange gefiederte und eine sehr feine, kurze Borste. Der eingliedrige Endopodit der 1. Maxille (Abb. 8) besitzt drei Borsten, der Exopodit ist durch eine Borste angedeutet. Die 2. Maxille (Abb. 10) zeigt im Bau keine abweichenden Besonderheiten. Der Maxilliped (Abb. 9) ist viergliedrig und insgesamt mit 6 z.T. abgespreizt gefiederten Borsten besetzt.

Alle Schwimmbeine sind zweigliedrig, die Dornformel der Außenastendglieder ist 3443 . Das 2. Basalglied des P1 (Abb. 11) trägt keinen Dorn. Die Form und Bewehrung des P2 und P3 (Abb. 12, 13) sind sehr ähnlich, die Dornen des Exopoditengliedes 2 erscheinen etwas kräftiger und schwach S-förmig gebogen, während sie am P3 etwas dünner und fast gerade ausgebildet sind. Die Dornen am Endglied des Exopoditen 4 (Abb. 14) sind gerade und ähnlich stark wie am P3. Alle Dornen sind gezähnt. Das Endglied des Endopoditen 4 (Abb. 15) ist etwa $1,75 \mathrm{mal}$ so lang wie breit, der einzige bestachelte Enddorn übertrifft die Gliedlänge etwas. Maße des Endgliedes Endopodit 4 und seines Enddorns sind: Länge : Breite $=40: 23 \mu \mathrm{m}=$ 1,74: 1; Enddorn $=42 \mu \mathrm{m}=1,05: 1$ auf die Gliedlänge bezogen. Die Verbindungsplatten der Schwimmbeine besitzen am P1 (Abb. 22) zwei seitliche, fast halbkreisförmige Vorwölbungen, am P2 (Abb. 23) ist die höchste Erhebung der Vorwölbungen laterad verlagert, außerdem inserieren in ihrem medianen Bereich einige längere Börstchen, am P3 (Abb. 24) sind die seitlichen Vorwölbungen weitgehend abgeflacht und tragen wenige in zwei Gruppen stehende Börstchen, am P4 (Abb. 25) ist der Distalrand der Verbindungsplatte nur noch schwach konkav, Börstchen fehlen. Vom ehemaligen Grundglied des P5 ist nur noch die Borste vorhanden, die auf dem 5. Thoraxsegment, etwas dorsad gestellt, inseriert. Das einzige freie Glied des P5 (Abb. 16) ist kurz, bis zu 1,5 mal so lang wie breit und trägt zwei an ihrer Basis etwa gleich starke Anhänge, von denen der laterale etwa dreimal so lang wie der mediale ist. Maße des P5 sind: Länge : Breite $=6: 5 \mu \mathrm{m}=1,2: 1$; medialer : lateraler Anhang $=7: 19 \mu \mathrm{m}=1: 2,71$. Die Borste des 5. Thoraxsegments ist $35 \mu \mathrm{m}$ lang. 


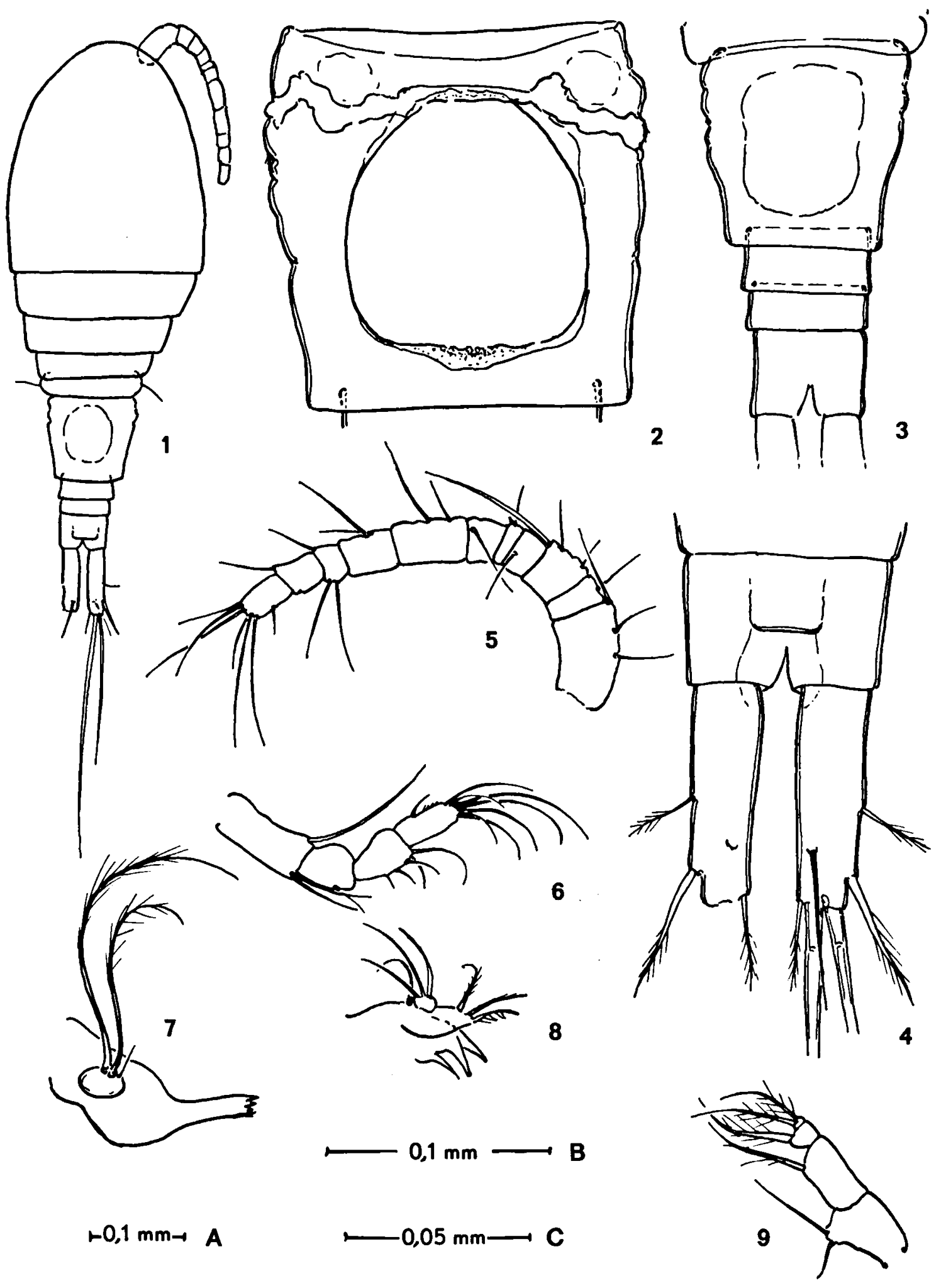

Abb. 1-9. Metacyclops agnitus n. sp., Q Holotypus: 1, Habitus (Maßstab A); 2, Genitalsegment (C); 3, Abdomen (B); 4, Analsegment und Furka (C); 5, 1. Antenne (B); 6, 2. Antenne (B); 7, Mandibel (C); 8, 1. Maxille (C); 9, Maxilliped (C). 
In den Eiersäcken der Weibchen wurden 4-9 Eier gezählt.

Zur eingehenderen Charakterisierung des Weibchens, besonders der Variabilität innerhalb der Population, sollen die nachstehenden absoluten und Verhältnismaße von 5 bzw. 6 Tieren dienen.

Länge von Thorax : Abdomen $=$

$\varnothing 360: 260 \mu \mathrm{m}$
$(343-381):(250-272)=(1,30-1,45: 1)$

Abdominalsegmente auf das Analsegment $=1$ berechnet $=$ $\varnothing 102: 31: 22: 39 \mu \mathrm{m}=$ $(98-106):(27-35):(21-23):(37-44)=$

$\varnothing 2,60: 0,79: 0,57: 1$

$(2,39-2,79):(0,68-0,89):(0,52-0,61): 1$

Furka, Länge : Breite =

$\varnothing(35+30): 19,5 \mu \mathrm{m}=(1,78+1,53): 1$

$(34-35+29-31):(19-21)=(1,67-1,84+1,43-1,63): 1$

Endborsten von außen nach innen $=$

$\varnothing 43,5: 153: 231$ : $26 \mu \mathrm{m}=$

$(42-45):(142-161):(222-240):(21-30)=$

$\varnothing 1: 3,52: 5,31: 0,60$

$\varnothing 1:(3,30-3,83):(4,93-5,58):(0,50-0,68)$

Dorsalborste $=\varnothing 41(46-44) \mu \mathrm{m}=1: 0,94(0,84-1,05)$

Lateralrandborste $=\varnothing 20(19-21) \mu \mathrm{m}=1: 0,46(0,42-$

$0,50)$, beide Borsten auf die laterale Apikalborste $=1$ berechnet.

Endglied Endopodit 4,

Länge : Breite $=\varnothing 38: 21 \mu \mathrm{m}=1,78: 1$ $(37-40):(20-23)=(1,71-1,95): 1$

Enddorn $=46(42-49) \mu \mathrm{m}=1,23(1,05-1,31): 1$ auf die Gliedlänge bezogen.

P5, Länge : Breite $=7(6-7): 5(4-6) \mu \mathrm{m}=1,34(1,17-$ $1,75): 1$

medialer : lateraler Anhang $=7(7-8): 21(19-24) \mu \mathrm{m}$ $=1: 2,91(2,71-3,14)$ auf den medialen Anhang berechnet.

Das Männchen wirkt in seinem Habitus durch einen schmaleren Thorax etwas schlanker als das Weibchen (Abb. 17). Die Länge des Thorax beträgt $293 \mu \mathrm{m}$, von denen $187 \mu \mathrm{m}$, also über $60 \%$ auf das Cephalothoraxsegment entfallen. An seinem Ende liegt mit $162 \mu \mathrm{m}$ auch die größte Breite des Thorax.

Das fünfgliedrige Abdomen ist $228 \mu \mathrm{m}$ lang, es besitzt im vorderen Drittel des kurzen Genitalsegments (Abb. 18) die größte Breite. Die Spermatophoren erscheinen in Aufsicht etwa kreisförmig. Das Analsegment ist auch beim Männchen länger als die beiden vorhergehenden Abdominalsegmente. Stellung und Form des Analoperculums gleichen denen des Weibchens. Absolute und Verhältnismaße des Abdomens sind:

Genitalsegm.

Länge : Breite Maße auf das Analsegm. $=1$ berechnet

Abd. segm. $\quad 49: 48 \mu \mathrm{m}=1,63: 1,09$

3. Abd. segm. $24: 45 \mu \mathrm{m}=0,80: 1,02$

4. Abd. segm. $19: 42 \mu \mathrm{m}=0,63: 0,95$

Analsegm.

$30: 44 \mu \mathrm{m}=1: 1$

Die Furkaläste sind geringfügig kürzer als beim Weibchen, die Verhältnismaße der Furkalborsten gleichen denen des Weibchens bis auf die Dorsalborste, die etwas länger ist. Die Maße der Furka und ihrer Anhänge sind = Länge : Breite $=(30+25): 17 \mu \mathrm{m}=(1,76+1,47)$ : 1; Endborsten von außen nach innen $=40$ : $145: 212: 26 \mu \mathrm{m}=1: 3,63: 5,30: 0,65$; Dorsalborste $=47 \mu \mathrm{m}=1,18: 1$ und Lateralrandborste $=19 \mu \mathrm{m}=0,48: 1$ (beide auf die laterale Apikalborste $=1$ bezogen).

Die Gliederung und Bewehrung der Greifantenne ist aus der Abbildung 21 zu erkennen. Das Endglied des Endopoditen 4 besitzt die folgenden Maße $=$ Länge $:$ Breite $=33: 17 \mu \mathrm{m}$ $=1,94: 1 ;$ Enddorn $=51 \mu \mathrm{m}=1,55: 1$ auf die Gliedlänge bezogen. Danach sind das Glied etwas gestreckter und der Enddorn länger als beim Weibchen. Die Maße des sehr kurzen P5 und seiner beiden Anhänge sind = Länge : Breite $=5: 4 \mu \mathrm{m}=1,25: 1$; innerer $:$ äußerer Anhang $=7: 21 \mu \mathrm{m}=1: 3,0$. Die rudimentäre Borste des Grundgliedes am 5. Thoraxsegment ist $40 \mu \mathrm{m}$ lang. Die Genitalklappenbewehrung (P6) besteht aus zwei Borsten, von denen die medial stehende mit $37 \mu \mathrm{m}$ über doppelt so lang wie die laterale mit $16 \mu \mathrm{m}$ ist (Abb. 19).

Abgesehen vom fünfgliedrigen Abdomen, der Greifantenne und der Genitalklappenbewehrung unterscheidet sich das Männchen geringfügig vom Weibchen durch die kürzere Furka, ihre längere Dorsalborste, dem schlankeren Endglied Endopodit 4 und seinem längeren Apikaldorn. 


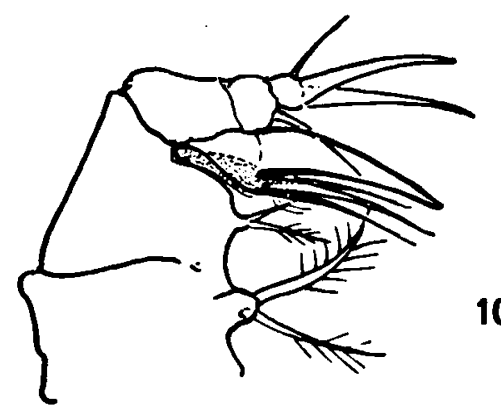

10
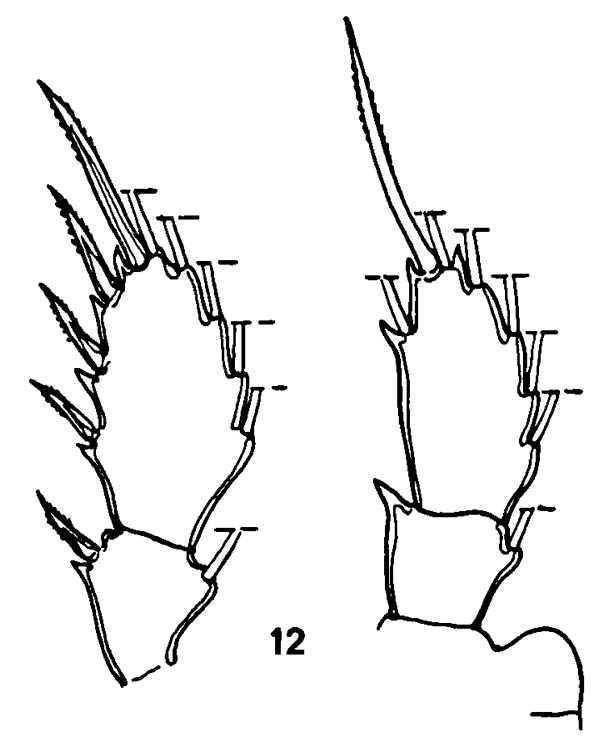

13
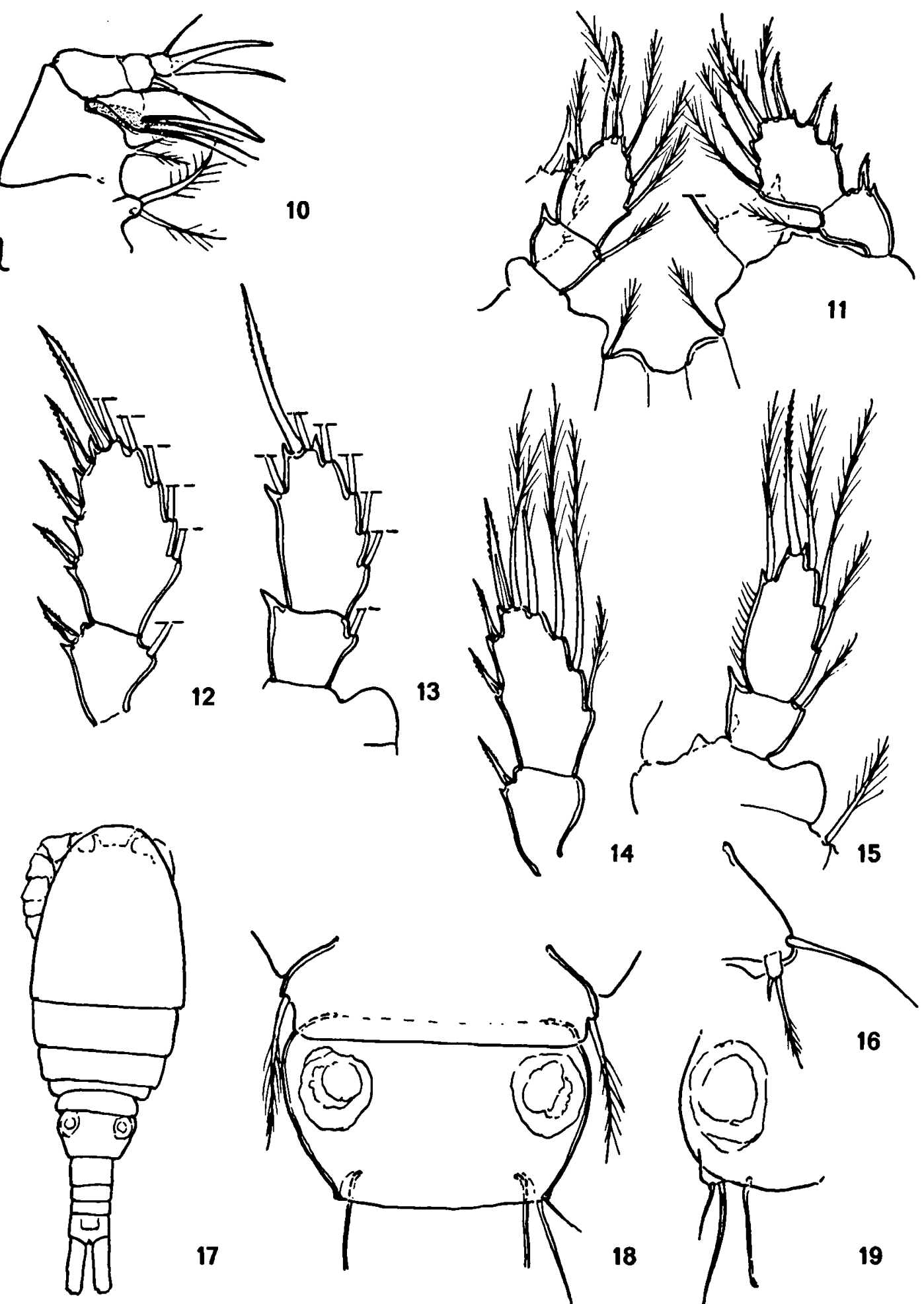

17

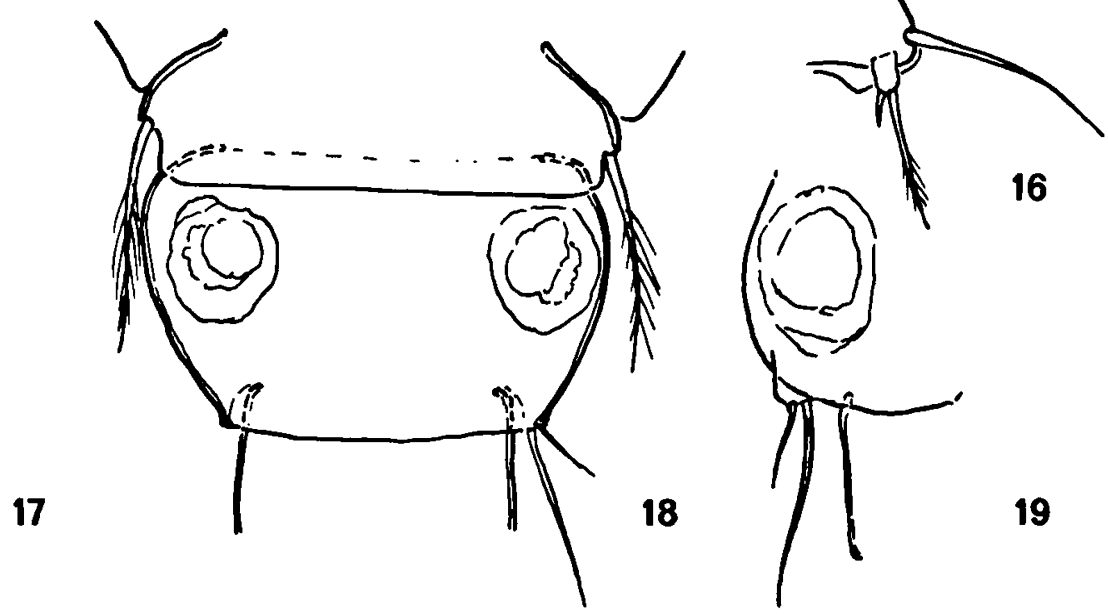

Abb. 10-19. Metacyclops agnitus n. sp. (10-16, \& Holotypus; 17-19, ơ Paratypus): 10, 2. Maxille (Maßstab C); 11, P1 (C); 12, Exopodit 3 (C); 13, Endopodit 3 (C); 14, Exopodit 4 (C); 15, Endopodit 4 (C); 16, P5 (C); 17, Habitus (A); 18, Genitalsegment (C); 19, Genitalklappenbewehrung (C). 
Verwandtschaftliche Beziehungen. Unter den Arten des Genus Metacyclops, die am Endglied des Endopoditen 4 nur einen Apikalanhang (Borste oder Dorn) tragen, besitzt Metacyclops agnitus $\mathrm{n}$. sp. die größte Ähnlichkeit mit dem altweltlichen Metacyclops minutus (Claus, 1863). Beschreibungen, Maßangaben und Abbildungen dieser Art liegen aus unterschiedlichen Regionen Europas, Asiens und Afrikas vor (Lindberg, 1936, 1940; Kiefer, 1937b, 1952; Dussart, 1967; Steib, 1985). Leider sind sie nicht so umfassend, daß ein Vergleich auf breiter Basis für alle taxonomisch wichtigen Merkmale angestellt werden kann. Möglicherweise verbergen sich unter diesen Metacyclops minutus noch nicht erkannte Spezies oder Subspezies.

Die sehr kurz gehaltene Originalbeschreibung von Claus (1863 : 102) und die drei kleinen Abbildungen (l.c., Taf. $\mathrm{X}, 6-8$ ) sind für einen eingehenderen Vergleich völlig unzureichend. Aus diesem Grunde wurden 10 Weibchen einer deutschen Population vermessen (Fundort: Tümpel am Wintermaar bei Disternich - Meßtischblatt Erp - etwa 30 km westlich von Bonn, leg. Herbst, 5. Juli 1965). Dabei wird von der Annahme ausgegangen, daß sich die deutschen Populationen der Art, zumindest in ihren Verhältnismaßen, nicht signifikant voneinander unterscheiden. Claus, l.c., gibt als Fundort "bei Cassel beobachtet" an.

Die durchschnittliche Gesamtlänge der Weibchen liegt bei $0,86(0,70-0,93) \mathrm{mm}$, der Thorax ist etwa doppelt so lang wie am Ende des Cephalothoraxsegments breit, Durchschnittsmaße sind $=526(476-585): 258(212-305) \mu \mathrm{m}=$ $1,97(1,83-2,27): 1$. Das Cephalothoraxsegment ist länger als die Thoraxsegmente 2-5 zusammengenommen.

Die Längen-Breiten-Maße der Abdominalsegmente sind: Genitalsegment $=128^{\prime}(123-130): 109(103-118)$ $\mu \mathrm{m}$; 2. Abdominalsegment $=47(43-53): 72(68-80) \mu \mathrm{m}$; 3. Abdominalsegment $=36(35-38): 66(62-69) \mu \mathrm{m}$; Analsegment $=42(39-46): 59(57-61) \mu \mathrm{m}$. Die Längen der Abdominalsegmente auf das Analsegment $=1$ bezogen sind demnach $=3,04(2,83-3,33): 1,11(1,02-1,26)$ : $0,86(0,76-0,95): 1$.

Maße der Furka und ihrer Anhänge sind: Länge : Breite $=46(44-48)+36(33-37): 23(21-24) \mu \mathrm{m}$, das entspricht einem Verhältnis von $1,99(1,91-2,14)+1,56(1,43-1,67)$ : 1 oder Gesamtlänge $=3,55(3,39-3,81): 1$. Die vier Endborsten besitzen von außen nach innen gesehen die folgenden absoluten Maße $=59(52-63): 164(154-175)$ : 244 (229-252) : 37 (35-41) $\mu \mathrm{m}$; auf die laterale Apikalborste $=1$ bezogen ergibt sich ein Verhältnis von $1: 2,78$ $(2,52-3,21): 4,16(3,88-4,58): 0,64(0,59-0,71)$. Das Längenverhältnis der beiden mittleren Endborsten untereinander ist relativ konstant, die Länge der lateralen von ihnen liegt bei $67 \%(64-70 \%)$ der medialen. Die Dorsalborste ist durchschnittlich 35,5 (33-39) $\mu \mathrm{m}$ lang $=0,60$ $(0,54-0,67)$ im Vergleich mit der lateralen Apikalborste. Die gleichen Maße der Lateralrandborste sind 24 (23-26) $\mu \mathrm{m}=0,41(0,39-0,48): 1$.

Das Endglied Endopodit 4 ist $48(45-50) \mu \mathrm{m}$ lang und 28 (26-30) $\mu \mathrm{m}$ breit, das entspricht einem LängenBreiten-Verhältnis von 1,72 (1,57-1,88) : 1. Der einzige Enddorn dieses Gliedes ist durchschnittlich 68,5 (64-72) $\mu \mathrm{m}$ lang, das ist zur Gliedlänge eine Verhältnis von 1,43 $(1,33-1,51): 1$.

Das einzige freie Glied des P5 ist 9 (9-10) $\mu \mathrm{m}$ lang und $5(4-6) \mu \mathrm{m}$ breit $=1,9(1,5-2,2): 1$. Die Länge des medialen zum lateralen Endanhang ist 7 (7-8) : 27 (24-30) $\mu \mathrm{m}$ $=1: 3,90(3,00-4,29)$.

Über diese Maßangaben hinaus können zur Charakteristik der Art die 11-gliedrige 1. Antenne, die zurückgelegt das Ende des Cephalothorax nicht erreicht, das unbedornte 2. Basalglied des P1 und die Ausbildung der Verbindungsplatten der Schwimmbeine (Abb. 2629) genannt werden. Unter ihnen sind am P1 die seitlichen Vorwölbungen am weitesten vorgezogen und unbewehrt, während sie an den drei folgenden Beinpaaren kontinuierlich flacher werden und - am geringsten am $\mathrm{P} 4-$ mit Härchen besetzt sind. Das sackförmige Receptaculum seminis ist in Höhe der Ausführungsgänge der Eileiter am breitesten (Abb. 30).

Bei den Männchen ist das Längenverhältnis der beiden Anhänge der Genitalklappenbewehrung zur Definition der Art wichtig. Bei drei Tieren der oben beschriebenen Population ist das Verhältnis vom inneren, schlanken Dorn zur äußeren Borste $=1,08,1,08$ und 1,13:1 (26 : 24 bzw. 26 : $23 \mu \mathrm{m}$ ). Die Längenverhältnisse der fünf Abdominalsegmente auf das Analsegment $=1$ bezogen sind $=2,00,1,97$, $1,91: 1,43,1,39,1,56: 1,06,1,06,1,09: 0,69$, $0,82,0,82: 1$. Offensichtlich ist die Dorsalborste der Furka länger als beim Weibchen $(0,81$, 0,88, 1,04: 1 auf die laterale Apikalborste bezogen) und das Endglied Endopodit 4 schlanker als dort (Länge : Breite $=2,13,2,14$ und 

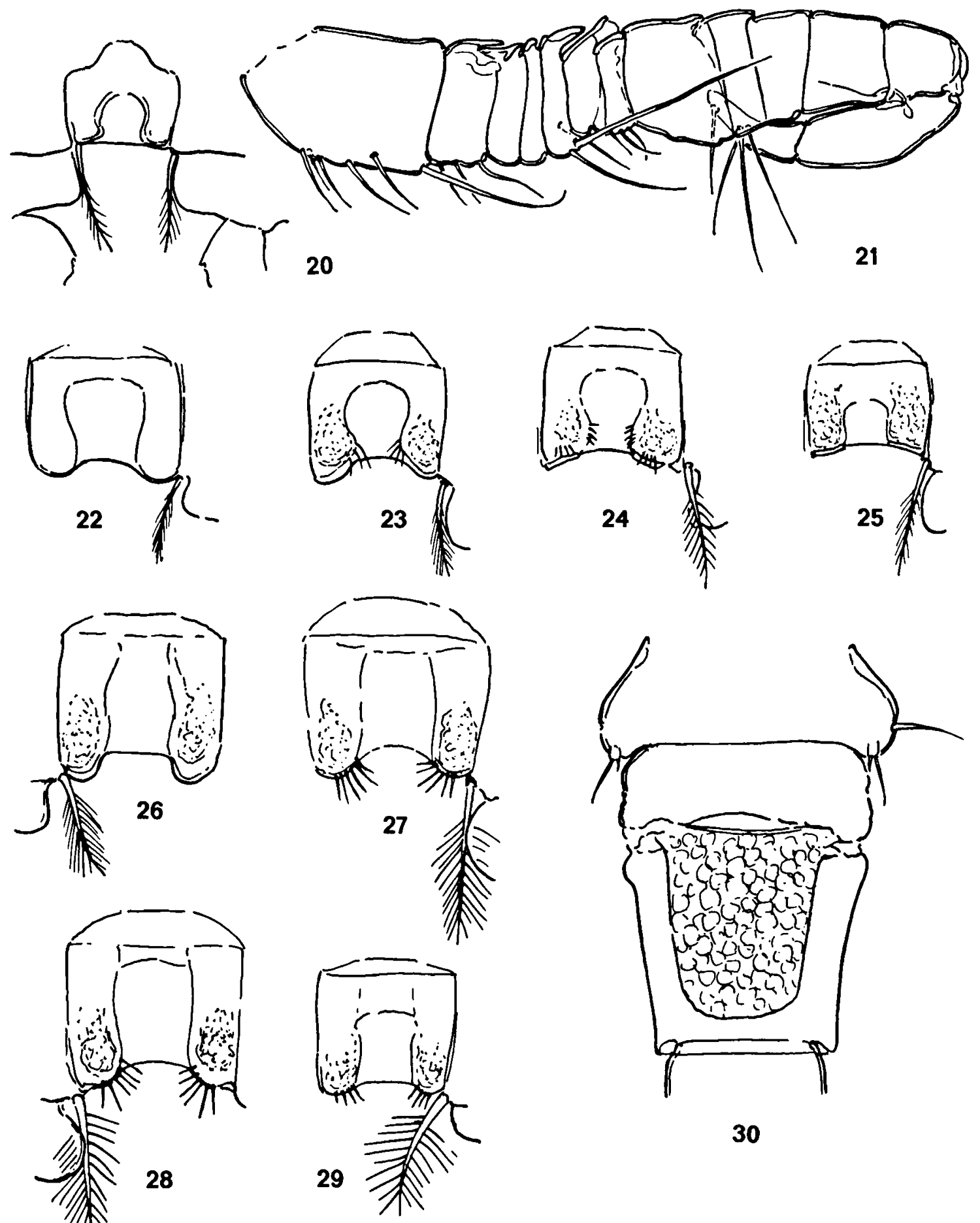

30

Abb. 20-25. Metacyclops agnitus n. sp. (20-21, ơ Paratypus; 22-25, \& Holotypus): 20, Verbindungsplatte P4 (Maßstab C); 21, 1. Antenne (C); 22, Verbindungsplatte P1 (C); 23, Verbindungsplatte P2 (C); 24, Verbindungsplatte P3 (C); 25, Verbindungsplatte P4 (C).

Abb. 26-30. Metacyclops minutus (Claus, 1863), ९: 26, Verbindungsplatte P1 (C); 27, Verbindungsplatte P2 (C); 28 , Verbindungsplatte P3 (C); 29, Verbindungsplatte P4 (C); 30, 5. Thoraxsegment und Genitalsegment (B). 
2,24 : 1). Die relative Länge des Enddorns vom Endglied Endopodit 4 im Verhältnis zur Gliedlänge übertrifft die beim Weibchen $(1,47,1,53$ und $1,56: 1$ ).

Nach den bisher bekannten Beschreibungen und den hier vorgelegten Maßen des sporadisch auftretenden Metacyclops minutus (Claus, 1863) unterscheidet sich Metacyclops agnitus n. sp. von ihm durch die Längenverhältnisse der Abdominalsegmente, die im Vergleich mit dem Analsegment kürzer sind, außerdem ist das Genitalsegment relativ breiter. Die Formen des Receptaculum seminis sind ebenfalls verschieden. Desweiteren weichen die Längenverhältnisse der Apikalborsten der Furka, speziell der beiden mittleren, mit $1: 3,5: 5,3: 0,6$ signifikant von denen des $M$. minutus ab. Die Form und Bewehrung der Verbindungsplatten der vier Schwimmbeinpaare erlaubt eine Trennung beider Arten. Schließlich ist der Längenunterschied der beiden Anhänge der Genitalklappenbewehrung des Männchens bei der neuen Art wesentlich größer als bei $M$. minutus $(2,3: 1$ gegen $1,1: 1$ ).

Metacyclops mutatus n. sp. (Abb. 31-48)

Material. - Holotypus: 19. Amsterdamer Expeditionen zu den Westindischen Inseln, Sta. 78-299, Aruba: Pos Chikito, Haus 74, geschlossener Brunnen mit einem Durchmesser unter $1 \mathrm{~m}$, Wasserspiegel in $2 \mathrm{~m}$ Tiefe, Wasserstand 0,2 m; Position 12 $27^{\prime} 58^{\prime \prime} \mathrm{N} 69^{\circ} 57^{\prime} 48^{\prime \prime} \mathrm{W}$; 18. Mai 1978; Paratypen 15 ९ᄋ, 18 ○’ $^{\circ}, 18$ Copepodide (Zoölogisch Museum Amsterdam).

Beschreibung. - Das Weibchen besitzt bei einer Gesamtlänge von $916 \mu \mathrm{m}$ ohne die Endborsten der Furka einen schlank cyclopoiden Habitus (Abb. 31). Die Thoraxlänge beträgt $545 \mu \mathrm{m}$, also etwa $3 / 5$ des gesamten Körpers. Seine größte Breite liegt mit $309 \mu \mathrm{m}=57 \%$ der Thoraxlänge, am Ende des Cephalothorax, dessen Länge wiederum mit $362 \mu \mathrm{m}$ rund $2 / 3$ des Thorax bildet.

Das Abdomen setzt sich aus einem robusten Genitalsegment (Abb. 32), das an den vorderen und mittleren Seiten stärkere Vorwölbungen aufweist, zwei kürzeren mittleren und dem län- geren Analsegment (Abb. 33) zusammen. Die Hinterränder aller Abdominalsegmente sind glatt, lediglich der Dorsalrand des Analsegments ist fein gezähnt. Das Receptaculum seminis (Abb. 32) ist fast kreisförmig ausgebildet und nimmt etwa die halbe Breite der ventralen Seite des Genitalsegments ein. Die Maße der Abdominalsegmente sind:

Länge : Breite Maße auf das Analsegm. = 1 berechnet (in Klammern Maße von zwei weiteren Weibchen)

Genitalsegm. $\quad 133: 134 \mu \mathrm{m}=2,61: 1,79$ $(2,69):(1,78)$

$(2,61):(1,70)$

2. Abd. segm. $45: 88 \mu \mathrm{m}=0,88: 1,17$

$(0,82):(1,17)$

$(0,93):(1,18)$

3. Abd. segm. $40: 81 \mu \mathrm{m}=0,78: 1,08$

$(0,71):(1,05)$

$(0,81):(1,05)$

Analsegm.

$51: 75 \mu \mathrm{m}=1: 1$

Die Furkaläste (Abb. 33) sind über $3^{1 / 2} \mathrm{mal}$ so lang wie in Höhe der Insertion der Lateralrandborste breit. Diese steht etwas hinter der Mitte des Seitenrandes (55-60\% der Furkalänge). Die Endborsten der Furka sind befiedert, die mediale ist etwas kürzer als die laterale, von den beiden mittleren erreicht die laterale etwa $3 / 4$ der Länge der medialen. Bei diesen beiden Borsten fallen die Verdickungen im proximalen Teil besonders auf (Abb. 35). Die Dorsalborste ist ungefähr so lang wie die laterale Apikalborste, ihre Insertion ist mediad verlagert. Die kurze Lateralrandborste wird vom Furkalast abgespreizt getragen. Die Maße der Furka und ihrer Anhänge sind:

$\begin{array}{ll}\text { Länge : Breite }=(56+46): 26 \mu \mathrm{m} & =(2,15+1,77): 1 \\ \text { Dito, zwei weitere Weibchen } & =(2,19+1,63): 1 \\ & =(2,07+1,43): 1 \\ \text { Endborsten von außen nach innen } & = \\ 55: 212: 278: 47 \mu \mathrm{m} & =1: 3,85: 5,05: 0,85 \\ \text { Dito, zwei weitere Weibchen } & =1: 3,34: 4,34: 0,98 \\ & =1: 3,30: 4,67: 0,75 \\ \text { Dorsalborste }=56 \mu \mathrm{m} & =1,02: 1 \\ \text { Dito, zwei weitere Weibchen } & =1,00: 1 \\ & =0,92: 1 \\ \text { Lateralrandborste }=30 \mu \mathrm{m} & =0,55: 1 \\ \text { Dito, zwei weitere Weibchen } & =0,57: 1 \\ & =0,52: 1\end{array}$


BIJDRAGEN TOT DE DIERKUNDE, 58 (1) - 1988

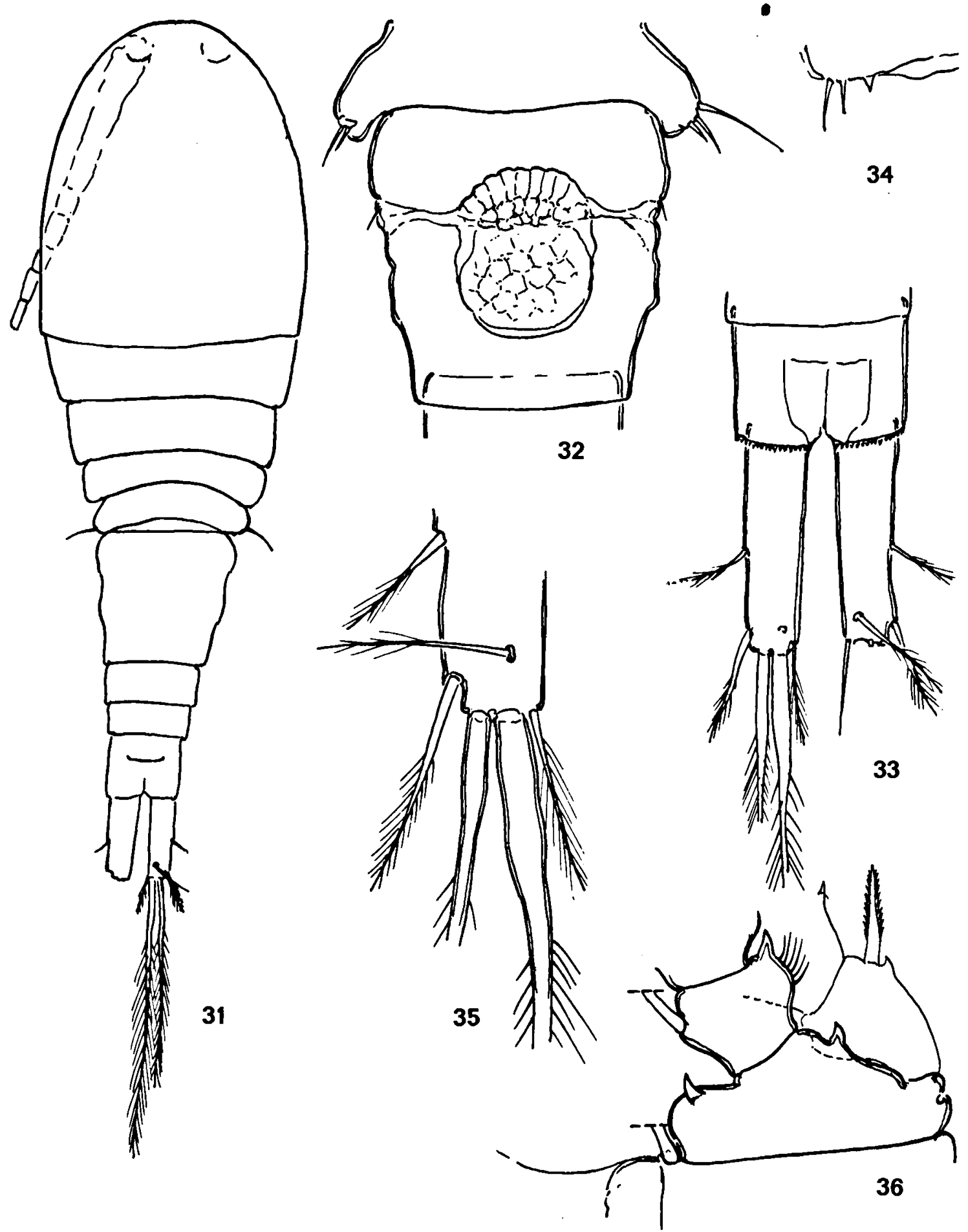

Abb. 31-36. Metacyclops mutatus n. sp., \& Holotypus: 31, Habitus (Maßstab A); 32, 5. Thoraxsegment und Genitalsegment (B); 33, Analsegment und Furka (B); 34, Genitalklappenbewehrung (C); 35, Ende des Furkalastes (C); $36,2$. Basalglied P3 (C). 
Die 11-gliedrige 1. Antenne (Abb. 37) reicht zurückgeschlagen bis zum Ende des Cephalothorax. Die mittleren Längenmaße der Glieder sind von der Basis an: $65: 18: 33: 16: 9: 24$ : $42: 39: 23: 28: 35 \mu \mathrm{m}$. Die viergliedrige 2 . Antenne (Abb. 38) trägt am vorletzten Glied 5, am letzten 7 Anhänge. Die Mandibel, die 1 . und 2. Maxille sowie der Maxilliped unterscheiden sich in ihrem Bau nicht von den gleichen Gliedmaßen des Metacyclops agnitus n. sp.

Die Schwimmbeine sind durchweg zweigliedrig, die Dornformel der Außenastendglieder ist 3443 . Die Verbindungsplatte des P1 besitzt beidseitig runde, glatte Vorwölbungen, das 2 . Basalglied trägt mediad keinen Dorn, das Endglied des Endopoditen ist terminal mit einem mächtigen, gekrümmten Dorn bewehrt (Abb. 39). Der 2. und 3. Schwimmfuß sind fast identisch gebaut, die Vorwölbungen der Verbindungsplatten (Abb. 40, 41) sind flacher als beim P1 und gehen gleitend in den Mittelteil über. Am nach innen vorgezogenen, gerundeten Teil des 2. Basalgliedes inseriert an beiden Schwimmfüßen ein laterad gekrümmter Dorn (Abb. 36). Die drei Dornen am Endglied des Exopoditen 4 sind schlanker als die vom P2 und P3 (Abb. 43). Alle Exopoditendorne sind gezähnt. Die Verbindungsplatte des P4 ähnelt in ihrem Bau der des P3. Das Endglied des Endopoditen 4 (Abb. 43) ist 1,5 mal so lang wie breit, der kräftige, mediale Enddorn ist um 1,4 mal länger als das Glied und über doppelt so lang wie der schwächere laterale Enddorn. Dieser schiebt sich über den medialen Dorn und ist deshalb schlechter zu erkennen und schwierig zu messen. Die Maße des Endgliedes Endopodit 4 und seiner Anhänge sind:

$\begin{array}{ll}\text { Länge : Breite }=45: 30 \mu \mathrm{m} & =1,50: 1 \\ \text { Dito, zwei weitere Weibchen } & =1,62: 1 \\ & =1,48: 1 \\ \text { med. : lat. Apikaldorn }=63: 26 \mu \mathrm{m} & =2,42: 1 \\ & =2,50: 1 \\ & =2,00: 1 \\ \text { Länge : med. Apikaldorn } & =1: 1,40 \\ \text { Dito, zwei weitere Weibchen } & =1: 1,38 \\ & =1: 1,43\end{array}$

Der P5 (Abb. 42) besteht nur noch aus einem flachen, breiten Glied, das mit zwei glatten, fast gleich starken Anhängen bewehrt ist. Der mediale erreicht etwa 2/3 der Länge des lateralen. Die lange Seitenrandborste des 5 . Thoraxsegments $(56 \mu \mathrm{m})$ ist als Rudiment des Basalgliedes vom P5 anzusehen. Maße des P5 und seiner Anhänge sind:

$\begin{array}{lll}\text { Länge : Breite }=7: 7 \mu \mathrm{m} & =1,0: 1 \\ \text { Dito, zwei weitere Weibchen } & =0,71: 1 \\ & =0,71: 1 \\ \text { med. : lat. Anhang }=16: 24 \mu \mathrm{m} & =1: 1,50 \\ \text { Dito, zwei weitere Weibchen } & =1: 1,31 \\ & & =1: 1,57\end{array}$

In der Probe wurde kein Weibchen mit vollständigen Eiersäckchen festgestellt.

Das Männchen besitzt einen geringfügig schlankeren Thorax als das Weibchen (Abb. 44), seine Maße sind: Länge : Breite $=398$ : $217 \mu \mathrm{m}$, demnach beträgt die Breite knapp $55 \%$ der Länge. Das $250 \mu \mathrm{m}$ lange Cephalothoraxsegment nimmt etwa $63 \%$ des Thorax ein.

Das fünfgliedrige Abdomen ist im Genitalsegment am breitesten. Die Spermatophoren erscheinen in Aufsicht breit oval. Die Maße der Abdominalsegmente sind:

Genitalsegm.

$$
\begin{aligned}
& \text { Länge : Breite Maße auf das Analsegm. } \\
& =1 \text { berechnet }
\end{aligned}
$$

: $108 \mu \mathrm{m}=1,71: 1,77$

2. Abd. segm. $\quad 50: 72 \mu \mathrm{m}=1,32: 1,18$

3. Abd. segm. $36: 65 \mu \mathrm{m}=0,95: 1,07$

4. Abd. segm. $29: 61 \mu \mathrm{m}=0,76: 1,00$

Analsegm. $\quad 38: 61 \mu \mathrm{m}=1: 1$

Abgesehen von etwas kürzeren Ästen und einer längeren Dorsalborste ähnelt die Furka der des Weibchens. Auch hier sind im basalen Abschnitt der beiden mittleren Furkalendborsten Verdickungen zu beobachten (Abb. 45). Maße der Furka und ihrer Anhänge sind: Länge : Breite $=(46+28): 23 \mu \mathrm{m}=(2,00$ $+1,22): 1$, Endborsten von außen nach innen $=56: 180: 261: 42 \mu \mathrm{m}=1: 3,21: 4,66: 075$; Dorsalborste $=68 \mu \mathrm{m}=1,21: 1$; Lateralrandborste $=29 \mu \mathrm{m}=0,52: 1$.

Der Bau der zum Greiforgan umgewandelten 1. Antenne ist aus der Abbildung 46 zu erkennen. Das Endglied Endopodit 4 und seine Anhänge besitzen die folgenden Maße: Länge : Breite $=41: 25 \mu \mathrm{m}=1,64: 1$; medialer $:$ late- 


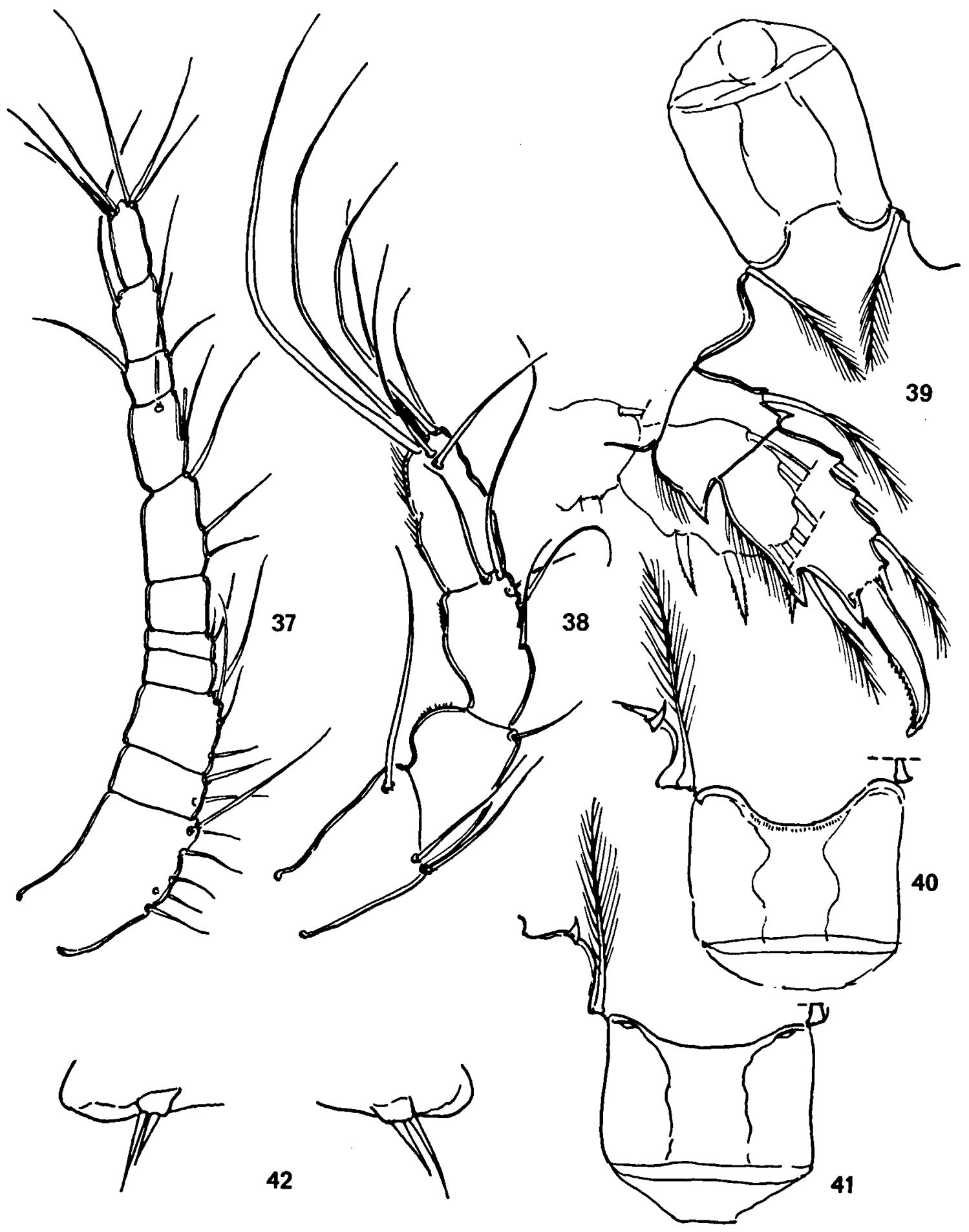

Abb. 37-42. Metacyclops mutatus n. sp., १ Holotypus: 37, 1. Antenne (Maßstab B); 38, 2. Antenne (C); 39, P1 (C); 40, Verbindungsplatte P2 (C); 41, Verbindungsplatte P3 (C); 42, beide P5 (C). 


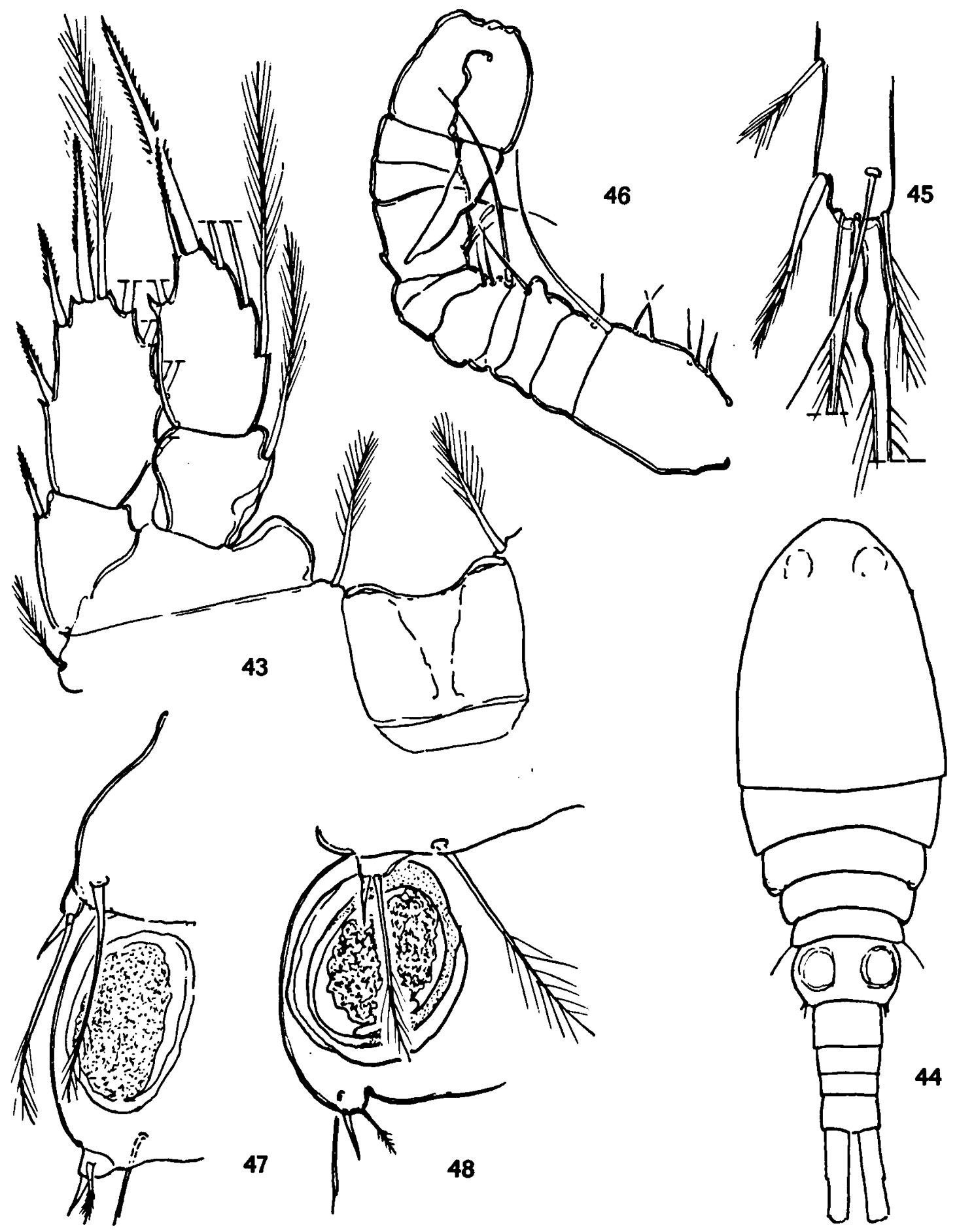

Abb. 43-48. Metacyclops mutatus n. sp. (43, ९ Holotypus, 44-48, ơ Paratypus): 43, P4 (Maßstab C); 44, Habitus (A); 45, Ende des Furkalastes (C): 46, 1. Antenne (B); 47, P5 und Genitalklappenbewehrung, ventral (C); 48, P5 und Genitalklappenbewehrung, lateral $(\mathrm{C})$. 
raler Enddorn $=58: 28 \mu \mathrm{m}=2,07: 1$; Länge $:$ medialer Enddorn $=1: 1,41$. Danach besteht in den Verhältnismaßen kein signifikanter Unterschied zu denen beim Weibchen. Auch beim Männchen besteht der P5 nur noch aus einem breiten Glied (Abb. 47, 48), das mit zwei hier schlanker erscheinenden Anhängen bewehrt ist, von denen die laterale, am Ende gefiederte Borste viel länger als beim Weibchen ist. Die Borste am 5. Thoraxsegment ist auch hier vorhanden und $58 \mu \mathrm{m}$ lang. Die Maße des P5 und seiner Anhänge sind: Länge : Breite = $6: 7 \mu \mathrm{m}=0,86: 1$, medialer Dorn : laterale Borste $=14: 50 \mu \mathrm{m}=1: 3,57$. Die Genitalklappenbewehrung (P6) besteht aus zwei relativ kurzen Anhängen, einem ventrad gestellten, glatten Dörnchen und einer dorsad daneben stehenden, fein gefiederten Borste, die in dieser Reihenfolge 15 und $20 \mu \mathrm{m}=1: 1,33$ lang sind (Abb. 47, 48).

Das Männchen unterscheidet sich vom Weibchen, wie üblich, durch das fünfgliedrige Abdomen, die Greifantenne und die unterschiedliche Ausbildung der Genitalklappenbewehrung. Bei $M$. mutatus n. sp. kommt, wie meistens, die kürzere Furka, besonders aber der wesentlich längere, laterale Apikalanhang des P5 hinzu.

Verwandtschaftliche Beziehungen. Unter den Metacyclops-Arten mit zwei Apikaldornen am Endglied des Endopodit 4, einer 11gliedrigen 1. Antenne und einer Furka, die etwa viermal so lang wie breit ist, kommen als Vergleichsarten $M$. aequatorialis Dussart, 1977 und $M$. chelazzi Dumont, 1981 infrage. Metacyclops mutatus n. sp. unterscheidet sich vom $M$. chelazzi durch den Bau des P5, durch die Form des Distalrandes der Verbindungsplatten der Schwimmbeine, die hier alle seitliche rundliche Vorwölbungen besitzen, das Vorhandensein eines mediad stehenden Dorns am 2. Basale des P1 und das Vorhandensein eines an gleicher Stelle stehenden Dorns am P4. Schließlich ist die Furka länger, ihre mediale Apikalborste und Dorsalborste relativ kürzer und der Längenunterschied der beiden mittleren Apikalborsten geringer als bei $M$. mutatus n. sp. M. aequa- torialis besitzt im Gegensatz zu der neuen Art an der Furka relativ kürzere Apikalborsten im Verhältnis zur lateralen Apikalborste, ein längeres Endglied Endopodit 4 und einen kürzeren medialen Apikaldorn an diesem Glied. Außerdem ist die laterale Borste am P5 länger und die Borste an der Seite des 5. Thoraxsegments kürzer. Nach dem Bau des P5 müßte $M$. mutatus n. sp. in die mendocinus-Verwandtschaft gehören, ist aber aufgrund seiner spezifischen Merkmale als eine neue Art anzusehen.

Obgleich bei manchen Artbeschreibungen die morphologischen Angaben lückenhaft sind, soll hier versucht werden, die bisher bekannten Metacyclops-Arten in einer Bestimmungstabelle zu erfassen. Sie soll als Arbeitsmittel bei künftigen Bestimmungen hilfreich sein. Außerdem kennzeichnet sie die Stellung der beiden neuen Metacylops-Arten im System.

BESTIMMUNGSSCHLÜSSEL FÜR DAS GENUS METACYCLOPS KIEFER, 1927

1. Endglied Endopodit 4 apikal mit 2 Anhängen bewehrt 19

- Endglied Endopodit 4 apikal mit einem Anhang.. 2

2. Apikaler Anhang des Endgliedes Endopodit 4 eine befiederte Borste, Furkaläste mit 3 Endborsten ...... M. trisetosus Herbst, 1957 (Europa, Jugoslawien)

- Apikaler Anhang des Endgliedes Endopodit 4 ein Dorn ............................................. 3

3. Erste Antenne 9-gliedrig. M. planus (Gurney, 1909) (Europa: 'Ischechoslowakei, Griechenland, Korfu, Bulgarien, Rumänien, Ungarn, Jugoslawien, Italien, Portugal, U.S.S.R.; Afrika: Marokko, Tunesien, Algerien, Aegypten, Sudan; Asien: Israel, Syrien, Iran)

- Erste Antenne 10-gliedrig.. M. amoenus Mann, 1940 (Asien, Türkei)

- Erste Antenne 11-gliedrig ........................ 4

4. Mediale Apikalborste der Furka länger als die laterale

— Mediale Apikalborste der Furka kürzer als die laterale

............................................... 8

5. Dorsalborste der Furka länger als die laterale Apikalborste

- Dorsalborste der Furka so lang wie die laterale Apikalborste .................................... 7

6. Dorsalborste doppelt so lang wie die laterale Apikalborste, Furkalast dreimal so lang wie breit........... M. subdolus Kiefer, 1938 
(Europa: Griechenland, Kreta, Italien, Sizilien, Sardinien, Balearen)

- Dorsalborste fast dreimal so lang wie die laterale Apikalborste der Furka, Längenverhältnis des Furkalastes 5,5-5,7 : 1 ............... gasparoi Stoch, 1987 (Europa: Italien)

7. Furka doppelt so lang wie breit, Endborste des P5 etwa viermal so lang wie der mediale Dorn. M. malayicus Kiefer, 1930 (Sumatra)

- Furka fast dreimal so lang wie breit, Endborste des P5 mindestens zehnmal so lang wie der winzige mediale Dorn .... M. curtispinosus Dussart, 1984 (Amerika: Venezuela)

8. Dornformel der Außenastendglieder der Schwimmbeine 3333 ... 9

- Dornformel der Außenastendglieder der Schwimmbeine 3443 .. 11

9. Der Dorn am Endglied Endopodit 4 ist länger als das Glied (etwa 1,35:1) .. M. monacanthus Kiefer, 1928 (Neuseeland)

- Der Dorn am Endglied Endopodit 4 ist kürzer als das Glied (etwa 0,75:1) .. 10

10. Endglied Endopodit 4 über zweimal so lang wie breit $(2,3: 1)$ M. arnaudi (Sars, 1908) (Australien)

- Endglied Endopodit 4 nicht doppelt so lang wie breit $(1,6: 1)$............. M. arnaudi platypus Kiefer, 1967 (Australien)

11. Furkaläste über viermal so lang wie breit ........ 12

- Furkaläste bis viermal so lang wie breit.......... 13

12. Apikaldorn des Endgliedes Endopodit 4 erreicht die Länge des Gliedes. M. grandispinifer Lindberg, 1940 (Asien: Türkei, Iran)

- Apikaldorn des Endgliedes Endopodit 4 viel länger als das Glied (etwa 1,6:1) ... M. prolatus Kiefer, 1935 (Afrika, Madagaskar)

13. Die Endborste am P5 ist bis zu viermal so lang wie der Dorn 14

- Die Endborste des P5 ist mindestens fünfmal länger als der Dorn 16

14. Enddorn am Endglied Endopodit 4 kürzer als das Glied M. lusitanus Lindberg, 1961 (Europa: Portugal)

- Enddorn am Endglied Endopodit 4 länger als das Glied. 15

15. Receptaculum seminis fast kreisrund, 2. und 3. Abdominalsegment kürzer als das Analsegment (0,75 : $0,55: 1)$, Genitalsegment bis $2,5 \mathrm{mal}$ so lang wie das Analsegment $M$. agnitus $\mathbf{n}$. sp. (Westindische Inseln: Barbados)

- Receptaculum seminis sackförmig, nur das 3. Abdominalsegment kürzer als das Analsegment $(0,8: 1)$, Genitalsegment über dreimal so lang wie das Analsegment. (Europa, Afrika, Asien)

16. Endglied Endopodit 4 über doppelt so lang wie breit, Furkaläste unter dreimal so lang wie breit, Dorn des
Endgliedes Endopodit 4 wenig länger als das Glied $(1,1: 1)$....................... concavus Kiefer, 1937 (Afrika: Angola)

- Endglied Endopodit 4 nicht doppelt so lang wie breit ...................................................... 17

17. Furka unter dreimal so lang wie breit $(2,7-2,8: 1)$, mittlere mediale Apikalborste der Furka etwa zehnmal so lang wie die laterale, Hinterränder aller Abdominalsegmente deutlich gezähnt.

M. denticulatus Dussart \& Frutos, 1985 (Amerika: Argentinien)

- Furka über dreimal so lang wie breit (3,4-3,5:1), mittlere mediale Apikalborste der Furka erreicht nicht die fünffache Länge der lateralen ........... 18

18. Längendifferenz der beiden mittleren Apikalborsten der Furka um 20\% .... M. communis Lindberg, 1938 (Asien: Indien)

- Längendifferenz der beiden mittleren Apikalborsten der Furka um 45\% .. M. pectiniatus Shen \& Tai, 1964 (Asien: Südchina)

19. Die beiden apikalen Anhänge des Endgliedes Endopodit 4 sind ein Dorn und eine gefiederte Borste....

(Europa: Mittelmeerraum)

- Beide Apikalanhänge des Endgliedes Endopodit 4 sind Dornen..................................... 20

20. Erste Antenne 17-gliedrig... M. grandis Kiefer, 1935 (Amerika: Uruguay)

- Erste Antenne 13-gliedrig M. tredecimus (Lowndes, 1934) (Amerika: Argentinien, Paraguay, Venezuela)

- Erste Antenne 12-gliedrig......................... 21

- Erste Antenne 11-gliedrig........................ 30

21. Furkaläste bis dreimal so lang wie breit .......... 22

- Furkaläste über dreimal so lang wie breit ........ 25

22. Mediale Furkalendborste länger als die laterale (1,6$1,8: 1) \ldots \ldots \ldots \ldots \ldots \ldots \ldots \ldots . . . \ldots$. brauni Herbst, 1962 (Amerika: Brasilien)

- Mediale Furkalendborste kürzer als die laterale.. 23

23. Furkaläste dreimal so lang wie breit, Verbindungsplatte P4 mit bedornten. seitlichen Vorwölbungen .. M. botosáneanui Pesce, 1985 (Westindische Inseln: Bonaire)

- Furkaläste zweimal so lang wie breit

24. Die beiden mittleren Endborsten der Furka untereinander wenig längenverschieden $(93: 100 \%)$, sie erreichen nicht die dreifache Länge der lateralen Apikalborste. M. hartmanni Herbst, 1960 (Amerika: Nicaragua)

- Der Längenunterschied der beiden mittleren Apikalborsten der Furka ist größer ( $80: 100 \%)$, sie erreichen die 4-5 fache Länge der lateralen Apikalborste

(Amerika: Paraguay) M. laticomis (Lowndes, 1934)

25. Furka 3-4 mal so lang wie breit

- Furka über viermal so lang wie breit ............. 27

26. Endborste des P5 über fünfmal länger als der mediale Dorn, Längendifferenz der beiden mittleren Apikal- 
borsten der Furka etwa $30 \%$

(Afrika: Südafrika)

$M$. necessarius (Kiefer, 1926)

- Endborste des P5 über zweimal länger als der mediale Dorn, Längendifferenz der beiden mittleren Apikalborsten um $10 \%$

M. mendocinus venezolanus (Kiefer, 1956) (als $M$. leptopus venezolanus Kiefer, 1956)

(Amerika: Venezuela, Brasilien)

27. Endglied Endopodit 4 über dreimal so lang wie breit, Längendifferenz der beiden mittleren Apikalborsten der Furka etwa 16-17\% 28

- Endglied Endopodit 4 höchstens 2,5 mal so lang wie breit, Längendifferenz der beiden mittleren Endborsten der Furka unter 10\% (4 bzw 8\%) .......... 29

28. Endborste des P5 über dreimal so lang wie der mediale Dorn ............... M. leptopus (Kiefer, 1927) (Amerika: Peru, Bolivien, Ecuador, Kolumbien)

- Endborste des P5 unter zweimal so lang wie der mediale Dorn. M. leptopus mucubajiensis Kiefer, 1956 (Amerika: Venezuela)

29. Medialer Enddorn des Endgliedes Endopodit 4 etwa doppelt so lang wie der laterale

M. mendocinus (Wierzejski, 1892)

(Amerika: Nicaragua, Kuba, Haiti, Puerto Rico, Ecuador, Peru, Venezuela, Kolumbien, Brasilien, Argentinien, Chile, Uruguay, Paraguay; Azoren)

- Medialer Enddorn des Endgliedes Endopodit 4 über 2,5 mal so lang wie der laterale.

M. problematicus Dumont, 1973 (Europa: Belgien)

30. Furka sechsmal so lang wie breit, medialer Apikaldorn am Endglied Endopodit 4 über 2,5 mal so lang wie der laterale............. somalicus Dumont, 1981 (Afrika: Somalia)

- Furka kürzer, bis etwa viermal so lang wie breit 31

31. Lateraler Apikaldorn am Endglied Endopodit $4 \mathrm{sehr}$ kurz (Verhältnis zum medialen etwa 1 : 5-7).... 32

- Lateraler Enddorn am Endglied Endopodit 4 länger (Verhältnis zum medialen bis $1: 2,5$ )........... 33

32. Dornformel 3333, mediale Endborste der Furka erreicht nicht die Länge der lateralen, Endglied Endopodit 4 knapp doppelt so lang wie breit......... M. trispinosus Dumont, $1981^{2}$ ) (Afrika: Guinea, Fouta Djalon)

- Dornformel 3443, mediale Endborste der Furka etwa 1,5 mal so lang wie die laterale, Endglied Endopodit 4 fast viermal so lang wie breit M. gracilis (Lilljeborg, 1853) (Europa; Asien: Türkei, Indien, Mongolei; Afrika: Senegal, Ruanda)

33. Furka etwa viermal so lang wie breit............ 34

- Furka kürzer...................................... 36

34. Endborste des P5 dreimal so lang wie der mediale Dorn, medialer Enddorn des Endgliedes Endopodit 4 kürzer als das Glied .. M. aequatorialis Dussart, 1978 (Afrika, Ruanda)
- Endborste des P5 weniger als doppelt so lang wie der mediale Dorn, medialer Enddorn des Endgliedes Endopodit 4 so lang oder länger als das Glied ... 35

35. Zweites Basale P1 an der inneren Vorwölbung mit Dorn, Dorsalborste der Furka kürzer als die laterale Apikalborste, laterale Borste des P5 gefiedert........ M. chelazzi Dumont, 1981 (Afrika, Somalia)

- Zweites Basale P1 ohne Dorn an der inneren Vorwölbung, Dorsalborste so lang wie die laterale Apikalborste, beide Anhänge des P5 unbefiedert.

(Westindische Inseln: Aruba)

M. mulatus n. sp.

36. Furka bis doppelt so lang wie breit, Endborste des P5 höchstens dreimal so lang wie der mediale Dorn 37

- Furka 2,7-3 mal so lang wie breit, Endborste des P5 5-10 mal länger als der mediale Dorn ............ 39

37. Mediale Apikalborste der Furka etwa doppelt so lang wie die laterale, Endglied Endopodit 4 über doppelt so lang wie breit (etwa 2,4:1), Endborste des P5 etwa dreimal so lang wie der mediale Dorn.

(Westindische Inseln: Haiti)

- Mediale Apikalborste der Furka kürzer als die laterale, Endglied Endopodit 41,5 mal so lang wie breit

38. Medialer Apikaldorn des Endgliedes Endopodit 4 so lang wie das Glied, lateraler Anhang des P5 eine befiederte Borste ............ M. micropus Kiefer, 1932 (Afrika: Elfenbeinküste)

- Medialer Apikaldorn des Endgliedes Endopodit 4 etwa 2/3 der Gliedlänge, lateraler Anhang des P5 eine starke, glatte Borste..... M. arenicolous Fryer, 1956 ${ }^{3}$ ) (Afrika: Malawi)

39. Die Endborste des P5 ist fünfmal so lang wie der Dorn .................................................... 40

- Diese Borste ist 8-10 mal so lang wie der Dorn.. 42

40. Genitalsegment mit seitlichen Vorwölbungen am Beginn des letzten Drittels, Verbindungsplatte P4 mit seitlichen Winkeln (Mesocyclops-ähnlich), Endglied Endopodit 4 nicht doppelt so lang wie breit .......... M. dentatus Pleşa, 1981

(als $M$. paludicola dentatus Pleșa, 1981)

(Westindische Inseln: Kuba)

- Genitalsegment ohne Vorwölbung, Verbindungsplatte P4 mit niedrigen seitlichen Wölbungen .... 41

41. Mediale Apikalborste der Furka länger als die laterale $(1,6: 1)$, Endglied Endopodit 4 reichlich doppelt so lang wie breit $(2,1: 1) \ldots \ldots \ldots . .$. . rudis Pleşa, 1981 (Westindische Inseln: Kuba)

- Mediale Apikalborste der Furka kürzer als die laterale $(0,9: 1)$, Endglied Endopodit 4 nur $1,5 \mathrm{mal}$ so lang wie breit ...................... campestris Reid, 1987 (Amerika: Zentral-Brasilien)

42. Mediale Apikalborste der Furka kürzer als die laterale (etwa $0,8: 1$ ) M. tropicus Kiefer, 1932 (Afrika: Elfenbeinküste) 
- Mediale Apikalborste der Furka länger als die laterale (etwa 1,3-1,5:1) 43

43. Die beiden Apikaldornen des Endgliedes Endopodit 4 sind gleichlang ........... subaequalis Dussart, 1984 (Amerika: Venezuela)

- Medialer Enddorn des Endgliedes Endopodit 4 etwa doppelt so lang wie der laterale $(1,8-2,1: 1)$ M. paludicola (Herbst, 1959) (als Cyclops (Microcyclops) paludicola Herbst, 1959) (Amerika: Brasilien)

\section{Bemerkungen zur Bestimmungstabelle}

1) Die Beurteilung der Synonymie des Metacyclops minutus (Claus, 1863) bereitet durch die oft auf wenige Merkmale begrenzten Angaben bei den Beschreibungen erhebliche Schwierigkeiten. In solchen Fällen kann die Identität nur vermutet werden, wie beispielsweise bei Cryptocyclops inopinatus Sars, 1927.

Absolute Maßangaben, aus denen möglichst auch die Variabilität einer Population hervorgeht, sind zur Absicherung ihrer Identität notwendig. Sie könnten die zur Zeit bestehenden Unsicherheiten beheben. So stellen der $M$. minutus senegalensis Gauthier, 1951 und der von Dussart, 1967 aus Spanien beschriebene $M$. minutus zwei untereinander ähnliche Formen dar. Sie sind besonders durch einen bedeutenden Längenunterschied zwischen Dorn und Borste des P5 gekennzeichnet (1: 7 bzw. 1 : 8 nach den Abbildungen), der bei $1: 3-4$ liegt.

Eine deutlich abweichende Population beschreibt Steib, 1985, aus Obervolta, deren Receptaculum seminis und das Längenverhältnis der Anhänge des P5 dem $M$. concavus ähnlich sind. Sie wird außerdem durch die im Verhältnis zum Analsegment kurzen Abdominalsegmente und den relativ kurzen Enddorn am Endglied Endopodit 4, der die Länge des Gliedes nur geringfügig überschreitet, charakterisiert. Bemerkenswert ist auch der geringe Längenunterschied der beiden mittleren Apikalborsten der Furka (13\%), der sonst wesentlich größer ist. Vermutlich handelt es sich um eine spezifische Art, deren Stellung nur durch absolute Maßangaben gesichert werden kann.

Schließlich sei auf den als Synonym zu $M$. minutus gestellten $M$. unacanthus (Lindberg, 1936) hingewiesen, dessen Abdominalsegmente 2 und 3 nach der Abbildung länger als das Analsegment sind und dessen Endglied Endopodit 4 doppelt so lang wie breit ist (nach der Abbildung 2,2:1).

2) Metacyclops trispinosus besitzt eine 11-gliedrige 1 . Antenne (briefliche Mitteilung von Prof. Dr. Dumont). Ob die Dornformel 3333 unter den Metacyclops-Arten mit 2 Apikalanhängen am Endglied Endopodit 4 einmalig ist, kann nicht mit absoluter Sicherheit gesagt werden, da bei den infrage kommenden 27 Arten die Dornformel nur $16 \mathrm{mal}$ genannt wurde.
3) Nach der Originalbeschreibung (Fryer, 1956: 226231) gibt es keinen Hinweis darauf, daß $M$. arenicolous in das Genus Bryocyclops zu stellen ist. Nach der Kennzeichnung des P5: "segment 2 reduced to a small sub-rectangular flap bearing two stout spiniform structures of which outermost is longest", handelt es sich offensichtlich um einen P5, der dem des oben beschriebenen $M$. mutatus n. sp. sehr ähnlich ist. Auch die übrigen Gliedmaßen und ihre Bewehrungen, z.B. 1. Antenne, Maxilliped, Gliederung und Zahl der Anhänge an den Schwimmbeinen sowie die Ausbildung des Analoperculums sind einwandfreie Beweise für die Gattungszugehörigkeit. Man sollte sich durch die etwas stilisierten Abbildungen nicht täuschen lassen und die Beurteilung der Form des einzigen Gliedes vom P5 nach dem Beschreibungstext ist Auffassungssache (vgl. Lindberg, 1961: 138).

\section{LITERATUR}

Claus, C., 1863. Die frei lebenden Copepoden mit besonderer Berücksichtigung der Fauna Deutschlands, der Nordsee und des Mittelmeeres: i-x, 1-230, Taf. IXXXVII (W. Engelmann, Leipzig).

Dumont, H. J., 1973. On Metacyclops problematicus, spec. nov., a new freshwater cyclopoid Copepod from Belgium, with a discussion of its taxonomic and ecological status. Zool. Anz., 191 (5/6): 329-337.

-, 1981 a. On a collection of zooplancton from Somalia with description of three new species of Copepoda. Monit. zool. ital., (N.S. 6), Suppl. 14 (7): 103-111.

- , 1981b. Cladocera and free-living Copepoda from the Fouta Djalon and adjacent mountain areas in West Africa. Hydrobiologia, 85: 97-116.

Dussart, B., 1967. Contribution à l'étude des Copépodes d'Espagne. Publicaciones Inst. Biol. apl. Barcelona, 42: 87-105.

- - 1978. Contribution à l'étude des Copépodes des eaux douces du Ruanda. Bull. Inst. fond. Afr. noire, (A) 39 (4): 821-840.

- - 1984. Some Crustacea Copepoda from Venezuela. Hydrobiologia, 113: 25-67.

Dussart, B. \&. Defaye, 1985. Répertoire mondial des Copépodes Cyclopoïdes: 1-236 (C.N.R.S., Paris).

Dussart, B. \&. M. Frutos, 1985. Sur quelques Copépodes d'Argentine. Rev. Hydrobiol. trop., 18 (4): 305-314.

Fryer, G., 1956. New species of cyclopoid and harpacticoid copepods from sandy beaches of Lake Nyasa. Ann. Mag. nat. Hist., (12) 9: 225-249.

Gauthier, H., 1951. Contributions à l'étude de la faune des eaux douces au Sénégal (Entomostracés): 1-169 (Imp. Minerva, Alger). 
Gurney, R., 1909. On the freshwater Crustacea from Algeria and Tunesia. Journ. roy. microsc. Soc. London, 1909: 273-305.

Herbst, H. V., 1957. Zoological results of a collecting journey to Yugoslavia, 1954. 5. Cyclopoida Gnathostoma (Crustacea Copepoda). Beaufortia, 5 (65): 223-240.

-—, 1959. Brasilianische Süßwassercyclopoiden (Crustacea Copepoda). Gewässer Abwässer, 24: 49-73.

- - 1960. Copepoden (Crustacea, Entomostraca) aus Nicaragua und Südperu. Gewässer Abwässer, 27: 27-54.

-, 1962 . Crustacea aus dem Amazonasgebiet, gesammelt von Professor Dr. H. Sioli und Dr. R. Braun, 1. Litorale und substratgebundene Cyclopoida Gnathostoma (Copepoda). Crustaceana, 3 (4): 259-278.

Kiefer, F., 1926. Diagnosen neuer SüßwasserCopepoden aus Afrika. Zool. Anz., 66 (9/12): 262-269.

-, 1927 . Beiträge zur Copepodenkunde (VI). Zool. Anz., 74 (5/6): 116-122.

- 1 1928. Beiträge zur Copepodenkunde (VIII). Zool. Anz., 76 (1/2): 5-18.

- - 1930. Neue Cyclopiden von den Sunda-Inseln. Zool. Anz., 90 (1/2): 55-58.

- 1 1932. Neue Diaptomiden und Cyclopiden aus Französisch-Westafrika. Bul. Soc. Şti. Cluj, 6: 523-528.

- 1933 . Freilebende Binnengewässercopepoden. Diaptomiden und Cyclopiden. In: Voyage de $\mathbf{C h}$. Alluaud et P. A. Chappuis en Afrique occidentale française. Arch. Hydrobiol., 26: 121-142.

- - 1934. Die freilebenden Copepoden Südafrikas. Zool. Jahrb., (Syst.) 65 (2): 99-192.

- , 1935a. Neue Süßwassercyclopiden (Crustacea Copepoda) aus Uruguay. Zool. Anz., 109 (7/8): 181-188.

- , 1935b. Neue Süßwassercyclopiden (Crustacea Copepoda) aus Ostafrika. Bul. Soc. Şti. Clüj, 8: 237-242.

- , 1937a. Die freilebenden Ruderfußkrebse (Crustacea Copepoda) Jugoslawiens. Glasn. skops. nauč. Društ. (= Bull. Soc. scient. Skoplje), (Sci. nat.) 18: 77-105.

-, $1937 \mathrm{~b}$. Contribution à l'étude du plancton d'eau douce d'Angola, II. Freilebende Ruderfußkrebse (Crustacea Copepoda) aus Angola, 1. Diaptomiden und Cyclopiden. Arch. Hydrobiol., 32: 470-485.

- , 1938. Cyclopiden (Crust. Cop.) aus süditalienischen Brunnen und Höhlen. Zool. Anz., 123 (1/2): 1-12.

- 1952 . Beitrag zur Kenntnis der Copepodenfauna Algeriens. Bull. Soc. Hist. nat. Afr. Nord, 43: 87-112.

- 1956 . Freilebende Ruderfußkrebse (Crustacea Copepoda), I. Calanoida und Cyclopoida. Ergebn. deutschen limnol. Venezuela-Exped. 1952, 1: 233-268.
- - 1967. Cyclopiden aus salzhaltigen Binnengewässern Australiens (Copepoda). Crustaceana, 12 (3): 292-302.

- , 1975. Freilebende Ruderfußkrebse (Crustacea Copepoda) des Titicacasees. Veröff. zool. Staatssamml. München, 4: 125-150.

Lilljeborg, W., 1853. Om de inom Skåne förekommande Crustaceer ordningarde Cladocera, Ostracoda och Copepoda. De crustaceis ex ordinibus tribus: Cladocera, Ostracoda et Copepoda, in Scania occurrentibus: i-xvi, 1-222, pls. I-XXVII (Berling, Lund).

LindBerg, K., 1936. Notes sur des Cyclopides (Crustacés Copépodes) de l'Iran. Bull. Mus. r. Hist. nat. Belg., 12 (17): 1-26.

- - 1938. Cyclopides (Crustacés Copépodes) nouveaux de l'Inde. Bull. Soc. zool. Fr., 63: 288-302.

- , 1940. Cyclopides (Crustacés Copépodes) de l'Inde, IV: Rec. Indian Mus., 42 (4): 567-588.

- , 1961. Remarques sur le genre Metacyclops (Kiefer 1927) et description d'un Metacyclops nouveau du Portugal. Kungl. fysiogr. Sällskap. Lund Förhandl., 31 (14): 133-145.

Lowndes, A. G., 1934. Reports of an expedition to Brazil and Paraguay in 1926-7 supported by the Trustees of the Percy Sladen Memorial Fund and the Executive Committees of the Carnegie Trust for Scotland. Copepoda. J. Linn. Soc. Lond., (Zool.) 39: 83-131.

ManN, A. K., 1940. Ueber pelagische Copepoden Türkischer Seen (mit Berücksichtigung des übrigen Plankton). Int. Rev. Hydrobiol., 40: 1-87.

Pesce, G. L., 1985. Amsterdam Expeditions to the West Indian Islands, Report 45. Cyclopids (Crustacea, Copepoda) from West Indian groundwater habitats. Bijdr. Dierk., 55 (2): 295-323.

Petkovski, T., 1963. Metacyclops mendocinus (Wierzejski) von Terceira - Azoren Inseln. Fragm. balcan., 5 (3): 13-18.

Pleșa, C., 1981. Cyclopides (Crustacea, Copepoda) de Cuba. Résult. Expéd. biospéol. cubano-roum. Cuba, 3: 17-34, 2 pls.

REID, J. W., 1987. The cyclopoid copepods of a wet campo marsh in central Brazil. Hydrobiologia, 153: 121-138.

SARs, G. O., 1908. Freshwater Copepoda from Victoria Southern Australia. Arch. Math. Naturv. Christiania, 29 (7): 1-24.

- , 1927. The freshwater Entomostraca of the Cape Province, Part 3: Copepoda. Ann. S. Afr. Mus., 25: 85-149, pls. V-XV.

Shen, C. J. A. Y. TAI, 1964. Descriptions of new species of freshwater Copepoda from Kwanghtung Province, South China. Acta zootaxon. Sinica, 1 (2): 367-396.

Steıв, K., 1985. Epidemiologie und Vekforökologie der Dracunculose in Obervolta (Burkina Faso), Westafrika: 1-279 (Diss. Univ. Hohenheim). 
Sтосн, F., 1987. Cave-dwelling cyclopoids (Crustacea, Copepoda) from Venezia Giulia (Northeastern Italy). Bull. zoöl. Mus. Univ. Amsterdam, 11 (5): 41-55.
WIERZEJSKI, A., 1892. Skornpiaki i wrotki (Rotatoria) slodkowodne zebrane w Argentynie. Rozpr. Akad. Umiejet. Kraków, 24: 222-246.

First draft received: 7 August 1987

Second draft received: 16 March 1988 